\title{
Cinchona Alkaloid-Lewis Acid Catalyst Systems for Enantioselective Ketene-Aldehyde Cycloadditions
}

\author{
Cheng Zhu, Xiaoqiang Shen and Scott G. Nelson* \\ Department of Chemistry, University of Pittsburgh, Pittsburgh, Pennsylvania 15260
}

\section{Supporting Information}

General Information: Optical rotations were measured on a Perkin-Elmer 241 digital polarimeter with a sodium lamp at ambient temperature and are reported as follows: $[\alpha]_{\lambda}(\mathrm{c} g / 100 \mathrm{~mL})$. Infrared spectra were recorded on a Nicolet Avatar 360 FT-IR spectrometer. NMR spectra were recorded on a Bruker Avance-300 $\left({ }^{1} \mathrm{H}: 300 \mathrm{MHz} ;{ }^{13} \mathrm{C}: 75 \mathrm{MHz}\right)$ spectrometer with chemical shifts reported relative to residual $\mathrm{CHCl}_{3}(7.27 \mathrm{ppm})$ for ${ }^{1} \mathrm{H}$ and $\mathrm{CDCl}_{3}(77.0 \mathrm{ppm})$ for ${ }^{13} \mathrm{C} \mathrm{NMR}$ spectra. Analytical high performance liquid chromatography (HPLC) was performed on a Hewlett Packard 1100 liquid chromatograph equipped with a variable wavelength UV detector (deuterium lamp, 190-600 nm) using a Daicel Chiracel ${ }^{\mathrm{TM}}$ OD-H column $(250 \times 4.6 \mathrm{~mm})$ (Daicel Inc.) or Daicel Chiralpak ${ }^{\mathrm{TM}} \mathrm{AD}$ column $(250 \mathrm{x} 4.6 \mathrm{~mm})$ (Daicel Inc.) and HPLC-grade isopropanol and hexanes as the eluting solvents. Analytical gas-liquid chromatography (GLC) was performed on a Varian 3900 gas chromatography equipped with a flame ionization detector and split mode capillary injection system using a Chiraldex ${ }^{\mathrm{TM}}$ G-TA column (20 m x $0.25 \mathrm{~mm}$ ) (Advanced Separation Technologies Inc.). Helium was used as the carrier gas at the indicated pressures. Unless otherwise stated, all reactions were carried out in dry glassware under a nitrogen atmosphere using standard inert atmosphere techniques for the manipulation of solvents and reagents. Anhydrous solvents $\left(\mathrm{CH}_{2} \mathrm{Cl}_{2}\right.$ and diethyl ether) were obtained by passage through successive aluminaand Q5 reactant-packed columns on a solvent purification system. $N, N$-Diisopropylethylamine was distilled under nitrogen from $\mathrm{CaH}_{2}$. All commercially available aldehydes were redistilled under $\mathrm{N}_{2}$. Commercially available acetyl chloride and propionyl chloride were redistilled under $\mathrm{N}_{2}$. Anhydrous $\mathrm{LiClO}_{4}$ (ReagentPlus) was purchased from Aldrich Chemical Co. and weighed out in a $\mathrm{N}_{2}$-filled glovebox. $O$-Trimethylsilylquinidine (TMSQ) and $O$-trimethylsilylquinine (TMS $q$ ) were prepared according to the literature procedure (TMSCl was added at $0^{\circ} \mathrm{C}$ for large scale preparations). ${ }^{1}$ Flash chromatography was performed as previously described on EM silica gel 60 (230-240 mesh). ${ }^{2}$ Characterization data for $\beta$-lactones $\mathbf{7 a}, \mathbf{b},{ }^{3} \mathbf{7} \mathbf{c}, \mathbf{d}^{4} \mathbf{7 e}, \mathbf{f}, \mathbf{j},{ }^{5}$ and $\mathbf{7} \mathbf{g}^{6}$ have been reported previously.

General procedure for asymmetric ketene-aldehyde cycloadditions: To a solution of TMS $Q$ (or TMS $q)(0.10 \mathrm{mmol})$ and $\mathrm{LiClO}_{4}(0.3-3.0 \mathrm{mmol})$ in $1.0 \mathrm{ml}$ of diethyl ether was added $2.0 \mathrm{ml}$ of $\mathrm{CH}_{2} \mathrm{Cl}_{2}$ and the reaction mixture was cooled to the indicated reaction temperature $\left(-78\right.$ or $\left.-40{ }^{\circ} \mathrm{C}\right)$. To the resulting mixture was added $0.44 \mathrm{ml}$ of $N, N$-diisopropylethylamine $(2.5 \mathrm{mmol})$ followed by the aldehyde $(1.0 \mathrm{mmol})$. A solution of $2.0 \mathrm{mmol}$ of acid chloride in $0.5 \mathrm{ml}$ of $\mathrm{CH}_{2} \mathrm{Cl}_{2}$ was then added over 1-4 h by syringe pump. The reaction mixture was stirred for 7-16 h then was quenched at the reaction temperature by adding $10 \mathrm{ml}$ of $\mathrm{Et}_{2} \mathrm{O}$ and the resulting mixture was filtered through silica gel eluting with $\mathrm{Et}_{2} \mathrm{O}(3 \times 20 \mathrm{~mL})$. The filtrate was concentrated in vacuo and the crude product mixture was purified by flash chromatography.

\footnotetext{
Calter, M. A. J. Org. Chem. 1996, 61, 8006-8007.

Still, W. C.; Kahn, M.; Mitra, A. J. Org. Chem. 1978, 43, 2923-2925.

Yang, H. W.; Romo, D. J. Org. Chem. 1997, 62, 4-5, supporting information.

Nelson, S. G.; Peelen, T. J.; Wan. Z. J. Am. Chem. Soc. 1999, 121, 9742-9743.

Nelson, S. G.; Zhu, C.; Shen, X. J. Am. Chem. Soc. 2004, 126, 14-15.

Nelson, S. G.; Wan, Z. Org. Lett. 2000, 2, 1883-1886.
} 


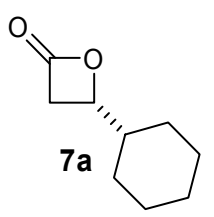

(S)-4-Cyclohexyloxetan-2-one (7a): The General Procedure was followed employing $40 \mathrm{mg}$ of TMSQ (0.10 mmol, $10 \mathrm{~mol} \%), 212 \mathrm{mg} \mathrm{L^{2 }}$, $\left.2.0 \mathrm{mmol}, 200 \mathrm{~mol} \%\right)$ and $120 \mu \mathrm{L}$ of cyclohexanecarboxaldehyde $(1.0 \mathrm{mmol})$ at a reaction temperature of $-40{ }^{\circ} \mathrm{C}$. Purification by flash chromatography (15\% diethyl ether in pentane) gave $131 \mathrm{mg}(85 \%)$ of the title compound as a colorless crystalline solid. Separation of the enantiomers by chiral GLC [Chiraldex ${ }^{\mathrm{TM}}$ G-TA column $20 \mathrm{~m}$ x $0.25 \mathrm{~mm}$, flow rate $0.5 \mathrm{~mL} / \mathrm{min}$, method: $100{ }^{\circ} \mathrm{C}$ for $10 \mathrm{~min}$, ramp @ 15.0 ${ }^{\circ} \mathrm{C} / \min$ to $130{ }^{\circ} \mathrm{C}$ for $8.0 \mathrm{~min}$, ramp @ $15.0{ }^{\circ} \mathrm{C} / \mathrm{min}$ to $160^{\circ} \mathrm{C}$ for $\left.10.0 \mathrm{~min}, \mathrm{~T}_{\mathrm{r}}: 24.5 \mathrm{~min}(4 S), \mathrm{T}_{\mathrm{r}}: 26.2 \mathrm{~min}(4 R)\right]$ provided the enantiomer ratio $(4 S):(4 R)=97: 3(94 \%$ ee $) .[\alpha]_{\mathrm{D}}+19.8\left(c 1.48, \mathrm{CHCl}_{3}\right)$.

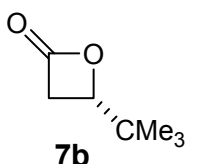

(S)-4-tert-Butyloxetan-2-one (7b): The General Procedure was followed employing $40 \mathrm{mg}$ of

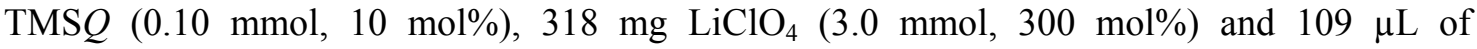
pivalaldehyde $(1.0 \mathrm{mmol})$ at a reaction temperature of $-78{ }^{\circ} \mathrm{C}$. Purification by flash chromatography (15\% diethyl ether in pentane) gave $91.3 \mathrm{mg}(71 \%)$ of the title compound as a colorless oil. Separation of the enantiomers by chiral GLC [Chiraldex ${ }^{\mathrm{TM}}$ G-TA column $20 \mathrm{~m} \times 0.25 \mathrm{~mm}$, flow rate $0.6 \mathrm{~mL} / \mathrm{min}$, method: $80^{\circ} \mathrm{C}$ for $5.0 \mathrm{~min}$, ramp @ $5.0{ }^{\circ} \mathrm{C} / \mathrm{min}$ to $100{ }^{\circ} \mathrm{C}$ for $10.0 \mathrm{~min}, \operatorname{ramp} @ 5.0{ }^{\circ} \mathrm{C} / \mathrm{min}$ to $130{ }^{\circ} \mathrm{C}$ for $5.0 \mathrm{~min}$, ramp @ $15.0{ }^{\circ} \mathrm{C} / \mathrm{min}$ to $150{ }^{\circ} \mathrm{C}$ for $5.0 \mathrm{~min}, \mathrm{~T}_{\mathrm{r}}: 17.8 \mathrm{~min}(4 R), \mathrm{T}_{\mathrm{r}}: 19.0 \mathrm{~min}(4 S)$ ] provided the enantiomer ratio $(4 S):(4 R)=98: 2(96 \%$ ee $) .[\alpha]_{\mathrm{D}}+20.7\left(c 2.00, \mathrm{CHCl}_{3}\right)$.

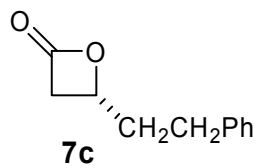

(R)-4-Phenethyloxetan-2-one (7c): The General Procedure was followed employing 40 $\mathrm{mg}$ of TMSQ $(0.10 \mathrm{mmol}, 10 \mathrm{~mol} \%), 53 \mathrm{mg} \mathrm{LiClO} 4(0.5 \mathrm{mmol}, 50 \mathrm{~mol} \%)$ and $132 \mu \mathrm{L}$ of hydrocinnamaldehyde $(1.0 \mathrm{mmol})$ at a reaction temperature of $-78{ }^{\circ} \mathrm{C}$. Purification by flash chromatography (25\% diethyl ether in pentane) gave $141 \mathrm{mg}(80 \%)$ of the title compound as a colorless oil. Separation of the enantiomers by chiral HPLC [Daicel Chiracel ${ }^{\mathrm{TM}}$ OD-H column, flow rate $1.0 \mathrm{~mL} / \mathrm{min}, 10 \%{ }^{i} \mathrm{PrOH}, 90 \%$ hexane, $\mathrm{T}_{\mathrm{r}} 15.5 \mathrm{~min}(S)$ and $17.3 \mathrm{~min}(R)$ ] provided the enantiomer ratio: $(R):(S)=96: 4(92 \%$ ee $) .[\alpha]_{\mathrm{D}}+48.9\left(\right.$ c $\left.1.20, \mathrm{CHCl}_{3}\right)$

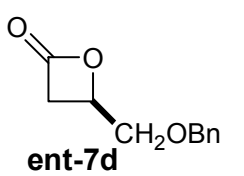

(R)-4-(Benzyloxy)methyl)oxetan-2-one (ent-7d): The General Procedure was followed

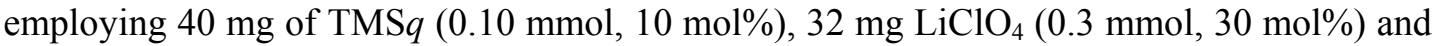
$140 \mu \mathrm{L}$ of benzyloxyacetaldehyde $(1.0 \mathrm{mmol})$ at a reaction temperature of $-78{ }^{\circ} \mathrm{C}$. Purification by flash chromatography (25\% ethyl acetate in hexane) gave $134 \mathrm{mg}(70 \%)$ of the title compound as a colorless oil. Separation of the enantiomers by chiral HPLC [Daicel Chiracel $^{\mathrm{TM}}$ OD-H column, flow rate $0.9 \mathrm{~mL} / \mathrm{min}, 15 \%{ }^{i} \mathrm{PrOH}, 85 \%$ hexane, $\mathrm{T}_{\mathrm{r}} 13.9 \mathrm{~min}(R)$ and $24.8 \mathrm{~min}(S)$ ] provided the enantiomer ratio: $(R):(S)=92: 8(84 \%$ ee $) .[\alpha]_{\mathrm{D}}-13.9\left(c 1.80, \mathrm{CHCl}_{3}\right)$.

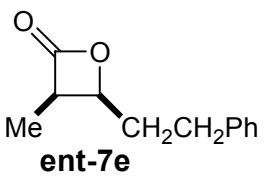

(3R, 4S)-3-Methyl-4-phenethyloxetan-2-one (ent-7e): The General Procedure was

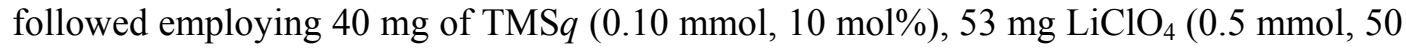
$\mathrm{mol} \%)$ and $132 \mu \mathrm{L}$ of hydrocinnamaldehyde $(1.0 \mathrm{mmol})$ at a reaction temperature of -78 ${ }^{\circ} \mathrm{C}$. Purification by flash chromatography $(10 \%$ ethyl acetate in hexane) gave $161 \mathrm{mg}$ $(84 \%)$ of the title compound as a colorless oil. Separation of enantiomers by Chiral HPLC [Daicel Chiracel ${ }^{\mathrm{TM}}$ OD-H column, flow rate $1.0 \mathrm{~mL} / \mathrm{min}, 5 \%{ }^{i} \mathrm{PrOH}, 95 \%$ hexane, $\mathrm{T}_{\mathrm{r}}: 12.9 \mathrm{~min}(3 S, 4 R), 14.0$ $\min (3 R, 4 S)]$ provided only one enantiomer $(3 R, 4 S)(>99 \%$ ee $)$. $[\alpha]_{\mathrm{D}}-47.2\left(c 2.04, \mathrm{CHCl}_{3}\right)$.

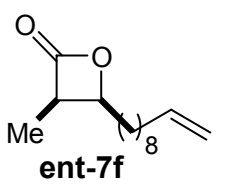

(3R, 4S)-4-(Dec-9-enyl)-3-methyloxetan-2-one (ent-7f): The General Procedure was followed employing $40 \mathrm{mg}$ of TMSq (0.10 mmol, $10 \mathrm{~mol} \%), 106 \mathrm{mg} \mathrm{\textrm {LiClO } _ { 4 }}$ (1.0 mmol, 100 mol\%) and $208 \mu \mathrm{L}$ of undecylenic aldehyde $(1.0 \mathrm{mmol})$ at a reaction temperature of $-78{ }^{\circ} \mathrm{C}$. Purification by flash chromatography (5\% ethyl acetate in hexane) gave $165 \mathrm{mg}$ (74\%) of the title compound as a colorless oil. Separation of enantiomers by chiral GLC [Chiraldex ${ }^{\text {TM }}$ G- 
TA column $20 \mathrm{~m} \times 0.25 \mathrm{~mm}$, flow rate $1.0 \mathrm{~mL} / \mathrm{min}$, method: $100{ }^{\circ} \mathrm{C}$ ramp $@ 10.0{ }^{\circ} \mathrm{C} / \mathrm{min}$ to $150{ }^{\circ} \mathrm{C}$ for 30.0 min, ramp@ $10.0{ }^{\circ} \mathrm{C} / \mathrm{min}$ to $160{ }^{\circ} \mathrm{C}$ for $10.0 \mathrm{~min}, \mathrm{~T}_{\mathrm{r}}: 29.2 \mathrm{~min}(3 S, 4 R) \mathrm{min}$ and $\left.30.0 \mathrm{~min}(3 R, 4 S)\right]$ provided the enantiomer ratio $(3 R, 4 S):(3 S, 4 R)=99.4: 0.6(99 \%$ ee $) .[\alpha]_{\mathrm{D}}-27.5\left(c 1.99, \mathrm{CHCl}_{3}\right)$.

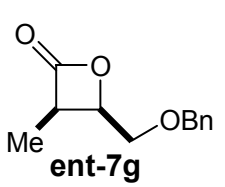

(3R, 4R)-4-(Benzyloxy)methyl)-3-methyloxetan-2-one (ent-7g): The General Procedure was followed employing $40 \mathrm{mg}$ of TMS $q(0.10 \mathrm{mmol}, 10 \mathrm{~mol} \%), 32 \mathrm{mg} \mathrm{\textrm {LClO } _ { 4 }}(0.3 \mathrm{mmol}$, $30 \mathrm{~mol} \%)$ and $140 \mu \mathrm{L}$ of benzyloxyacetaldehyde $(1.0 \mathrm{mmol})$ at a reaction temperature of $-78{ }^{\circ} \mathrm{C}$. Purification by flash chromatography $(20 \%$ ethyl acetate in hexane) gave $140 \mathrm{mg}$ (68\%) of the title compound as a colorless oil. Separation of the enantiomers by chiral HPLC [Daicel Chiracel ${ }^{\mathrm{TM}}$ OD-H column, flow rate $1.0 \mathrm{~mL} / \mathrm{min}, 10 \%{ }^{i} \mathrm{PrOH}, 90 \%$ hexane, $\mathrm{T}_{\mathrm{r}} 13.4 \mathrm{~min}(3 R, 4 R)$ and $31.4 \min (3 S, 4 S)]$ provided the enantiomer ratio: $(3 R, 4 R):(3 S, 4 S)=99.5: 0.5(99 \%$ ee $) . \quad[\alpha]_{\mathrm{D}}-7.6(c 1.58$, $\left.\mathrm{CHCl}_{3}\right)$.

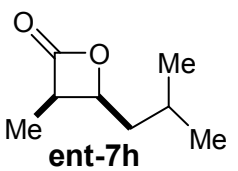

(3R, 4S)-4-Isobutyl-3-methyloxetan-2-one (ent-7h): The General Procedure was followed employing $40 \mathrm{mg}$ of $\mathrm{TMS} q(0.10 \mathrm{mmol}, 10 \mathrm{~mol} \%), 212 \mathrm{mg} \mathrm{\textrm {LiClO } _ { 4 }}(2.0 \mathrm{mmol}, 200 \mathrm{~mol} \%)$ and $109 \mu \mathrm{L}$ of isovaleraldehyde $(1.0 \mathrm{mmol})$ at a reaction temperature of $-78{ }^{\circ} \mathrm{C}$. Purification by flash chromatography (20\% diethyl ether in pentane) gave $103 \mathrm{mg}(72 \%)$ of the title compound as a colorless oil. Separation of enantiomers by chiral GLC [Chiraldex TM G-TA column 20 $\mathrm{m} \times 0.25 \mathrm{~mm}$, flow rate $0.6 \mathrm{~mL} / \mathrm{min}$, method: $80{ }^{\circ} \mathrm{C}$ for $5.0 \mathrm{~min}$, ramp $@ 5.0{ }^{\circ} \mathrm{C} / \mathrm{min}$ to $100{ }^{\circ} \mathrm{C}$ for $10.0 \mathrm{~min}$, ramp@ $9.0{ }^{\circ} \mathrm{C} / \mathrm{min}$ to $130{ }^{\circ} \mathrm{C}$ for $5.0 \mathrm{~min}, \mathrm{~T}_{\mathrm{r}}: 23.9 \mathrm{~min}(3 R, 4 S)$ and $\left.25.0 \mathrm{~min}(3 S, 4 R)\right]$ provided the enantiomer ratio $(3 R, 4 S):(3 S, 4 R)=99.7: 0.3\left(99.4 \%\right.$ ee). $[\alpha]_{\mathrm{D}}-41.8\left(c 1.88, \mathrm{CHCl}_{3}\right)$. IR (thin film): $1824,1465,1124 \mathrm{~cm}^{-}$ ${ }^{1}$; ${ }^{1} \mathrm{H}$ NMR $\left(300 \mathrm{MHz}, \mathrm{CDCl}_{3}\right.$ ) $\delta 4.67$ (ddd, J = 9.7, 6.5, $\left.3.8 \mathrm{~Hz}, 1 \mathrm{H}\right), 3.77(\mathrm{qd}, \mathrm{J}=7.8,6.5 \mathrm{~Hz}, 1 \mathrm{H}), 1.82(\mathrm{~m}$, $1 \mathrm{H}), 1.71(\mathrm{ddd}, \mathrm{J}=14.3,9.7,6.0 \mathrm{~Hz}, 1 \mathrm{H}), 1.51(\mathrm{ddd}, \mathrm{J}=14.3,7.6,3.8 \mathrm{~Hz}, 1 \mathrm{H}), 1.29(\mathrm{~d}, \mathrm{~J}=7.8 \mathrm{~Hz}, 3 \mathrm{H}), 1.01$ $(\mathrm{d}, \mathrm{J}=6.6 \mathrm{~Hz}, 3 \mathrm{H}), 0.99(\mathrm{~d}, \mathrm{~J}=6.6 \mathrm{~Hz}, 3 \mathrm{H}) ;{ }^{13} \mathrm{C} \mathrm{NMR}\left(75 \mathrm{MHz}, \mathrm{CDCl}_{3}\right) \delta 172.7,74.2,47.4,38.5,25.2,22.8$, 22.0, 8.1; Anal. calcd for $\mathrm{C}_{8} \mathrm{H}_{14} \mathrm{O}_{2}$ : C, 67.57; H, 9.92; found: $\mathrm{C}, 67.44 ; \mathrm{H}, 10.20$.

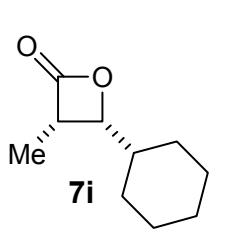

(3S, 4R)-4-Cyclohexyl-3-methyloxetan-2-one (7i): The General Procedure was followed

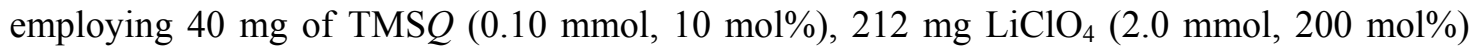
and $120 \mu \mathrm{L}$ of cyclohexanecarboxaldehyde $(1.0 \mathrm{mmol})$ at a reaction temperature of $-40{ }^{\circ} \mathrm{C}$. Purification by flash chromatography (15\% diethyl ether in pentane) gave $124 \mathrm{mg}$ (74\%) of the title compound as a colorless crystalline solid. Separation of enantiomers by chiral GLC [Chiraldex ${ }^{\text {TM }}$ G-TA column $20 \mathrm{~m}$ x $0.25 \mathrm{~mm}$, flow rate $0.5 \mathrm{~mL} / \mathrm{min}$, method: $100{ }^{\circ} \mathrm{C}$ for $10 \mathrm{~min}$, ramp @ 15.0 ${ }^{\circ} \mathrm{C} / \mathrm{min}$ to $130{ }^{\circ} \mathrm{C}$ for $8.0 \mathrm{~min}$, ramp @ $15.0{ }^{\circ} \mathrm{C} / \mathrm{min}$ to $160{ }^{\circ} \mathrm{C}$ for $15.0 \mathrm{~min}, \mathrm{~T}_{\mathrm{r}}: 26.8 \mathrm{~min}(3 \mathrm{~S}, 4 R), \mathrm{T}_{\mathrm{r}}: 27.7 \mathrm{~min}$ $(3 R, 4 S)]$ provided the enantiomer ratio $(3 S, 4 R):(3 R, 4 S)=98.5: 1.5(97 \%$ ee $)$. $[\alpha]_{\mathrm{D}}-13.4\left(c 1.45, \mathrm{CHCl}_{3}\right)$. IR (thin film): 2932, 2854, 1825, 1132 $\mathrm{cm}^{-1} ;{ }^{1} \mathrm{H}$ NMR $\left(300 \mathrm{MHz}, \mathrm{CDCl}_{3}\right) \delta 4.18(\mathrm{dd}, \mathrm{J}=10.6,6.3 \mathrm{~Hz}, 1 \mathrm{H}), 3.72$ $(\mathrm{qd}, \mathrm{J}=7.8,6.3 \mathrm{~Hz}, 1 \mathrm{H}), 2.01-1.92(\mathrm{~m}, 1 \mathrm{H}), 1.82-1.52(\mathrm{~m}, 5 \mathrm{H}), 1.34(\mathrm{~d}, \mathrm{~J}=7.8 \mathrm{~Hz}, 3 \mathrm{H}), 1.32-1.19(\mathrm{~m}, 3 \mathrm{H})$, 1.07-0.88 (m, 2H); ${ }^{13} \mathrm{C}$ NMR $\left(75 \mathrm{MHz}, \mathrm{CDCl}_{3}\right) \delta 172.9,79.0,46.8,37.7,28.9,28.1,26.0,25.0$ (2C), 8.4; HRMS $m / z$ calcd for $\mathrm{C}_{10} \mathrm{H}_{16} \mathrm{O}_{2}: 168.1150$; found: 168.1158 .

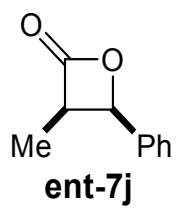

(3R, 4R)-3-Methyl-4-phenyloxetan-2-one (ent-7j): The General Procedure was followed

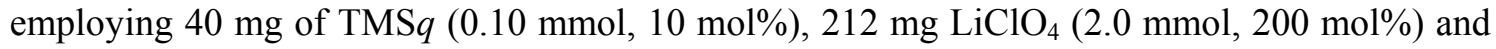
$102 \mu \mathrm{L}$ of benzaldehyde $(1.0 \mathrm{mmol})$ at a reaction temperature of $-78{ }^{\circ} \mathrm{C}$. Purification by flash chromatography (10\% ethyl acetate in hexane) gave $126 \mathrm{mg}(78 \%)$ of the title compound as a colorless oil. Separation of the enantiomers by chiral HPLC [Daicel Chiracel ${ }^{\mathrm{TM}}$ OD-H column, flow rate $1.0 \mathrm{~mL} / \mathrm{min}, 3 \%{ }^{i} \mathrm{PrOH}, 97 \%$ hexane, $\mathrm{T}_{\mathrm{r}} 10.8 \mathrm{~min}(3 R, 4 R), 11.7 \mathrm{~min}(3 S, 4 S)$ ] provided only one enantiomer $(3 R, 4 R)(>99 \%$ ee $) .[\alpha]_{\mathrm{D}}+139\left(c 1.79, \mathrm{CHCl}_{3}\right)$. 


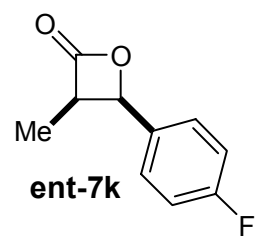

(3R, 4R)-4-(4-Fluorophenyl)-3-methyloxetan-2-one (ent-7k): The General Procedure was followed employing $40 \mathrm{mg}$ of $\mathrm{TMS} q(0.10 \mathrm{mmol}, 10 \mathrm{~mol} \%), 212 \mathrm{mg} \mathrm{LiClO}_{4}(2.0$ mmol, $200 \mathrm{~mol} \%)$ and $107 \mu \mathrm{L}$ of 4-fluorobenzaldehyde $(1.0 \mathrm{mmol})$ at a reaction temperature of $-78{ }^{\circ} \mathrm{C}$. Purification by flash chromatography $(20 \%$ ethyl acetate in hexane) gave $153 \mathrm{mg}(85 \%)$ of the title compound as a colorless oil. Separation of enantiomers by chiral HPLC [Daicel Chiralpak ${ }^{\mathrm{TM}} \mathrm{AD}$ column, flow rate $1.0 \mathrm{~mL} / \mathrm{min}, 5 \%$ ${ }^{i} \mathrm{PrOH}, 95 \%$ hexane, $\left.\mathrm{T}_{\mathrm{r}} 9.0 \mathrm{~min}(3 \mathrm{~S}, 4 S), 9.7 \mathrm{~min}(3 R, 4 R)\right]$ provided only one enantiomer $(3 R, 4 R)(>99 \%$ ee $)$. $[\alpha]_{\mathrm{D}}+124\left(c 1.03, \mathrm{CHCl}_{3}\right)$; IR (thin film): 1828, 1510, 1228 $\mathrm{cm}^{-1} ;{ }^{1} \mathrm{H}$ NMR $\left(300 \mathrm{MHz}, \mathrm{CDCl}_{3}\right) \delta 7.30(\mathrm{~m}, 2 \mathrm{H})$, 7.13 (t J = 8.6 Hz, 2H), $5.65(\mathrm{~d}, \mathrm{~J}=6.4 \mathrm{~Hz}, 1 \mathrm{H}), 4.06(\mathrm{qd}, \mathrm{J}=7.8,6.4 \mathrm{~Hz}, 1 \mathrm{H}), 0.95(\mathrm{~d}, \mathrm{~J}=7.8 \mathrm{~Hz}, 3 \mathrm{H}) ;{ }^{13} \mathrm{C}$ $\operatorname{NMR}\left(75 \mathrm{MHz}, \mathrm{CDCl}_{3}\right) \delta 171.8,162.7(\mathrm{~d}, \mathrm{~J}=246 \mathrm{~Hz}), 130.4(\mathrm{~d}, \mathrm{~J}=2.9 \mathrm{~Hz}), 127.5(\mathrm{~d}, \mathrm{~J}=8.3 \mathrm{~Hz}), 115.6(\mathrm{~d}, \mathrm{~J}=$ $21.7 \mathrm{~Hz}), 74.6,50.1,9.4$; HRMS $m / z$ calcd for $\mathrm{C}_{10} \mathrm{H}_{9} \mathrm{FO}_{2}$ : 180.0587; found: 180.0592 .

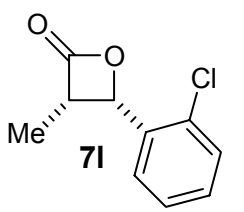

(3S, 4S)-4-(2-Chlorophenyl)-3-methyloxetan-2-one (71): The General Procedure was followed employing $40 \mathrm{mg}$ of TMSQ $(0.10 \mathrm{mmol}, 10 \mathrm{~mol} \%), 212 \mathrm{mg} \mathrm{\textrm {LiClO } _ { 4 }}(2.0 \mathrm{mmol}$, $200 \mathrm{~mol} \%)$ and $113 \mu \mathrm{L}$ of 2 -chlorobenzaldehyde $(1.0 \mathrm{mmol})$ at a reaction temperature of $-78{ }^{\circ} \mathrm{C}$. Purification by flash chromatography $(10 \%$ diethyl ether in pentane) gave $158 \mathrm{mg}$ $(80 \%)$ of the title compound as a colorless oil. Separation of enantiomers by chiral HPLC [Daicel Chiracel ${ }^{\mathrm{TM}}$ OD-H column, flow rate $0.6 \mathrm{~mL} / \mathrm{min}, 2 \%{ }^{i} \mathrm{PrOH}, 98 \%$ hexane, $\mathrm{T}_{\mathrm{r}} 13.4 \mathrm{~min}(3 R, 4 R), 14.1$ min $(3 S, 4 S)]$ provided only one enantiomer $(3 S, 4 S)\left(>99 \%\right.$ ee). $[\alpha]_{\mathrm{D}}-201\left(c\right.$ 1.68, $\left.\mathrm{CHCl}_{3}\right)$; IR (thin film): $2979,1827,1472,1444,1289,1055,946,880,755 \mathrm{~cm}^{-1} ;{ }^{1} \mathrm{H}$ NMR $\left(300 \mathrm{MHz}, \mathrm{CDCl}_{3}\right) \delta 7.55-7.52(\mathrm{~m}, 1 \mathrm{H}), 7.43-$ $7.31(\mathrm{~m}, 3 \mathrm{H}), 5.86(\mathrm{~d}, \mathrm{~J}=6.3 \mathrm{~Hz}, 1 \mathrm{H}), 4.16(\mathrm{qd}, \mathrm{J}=7.7,6.3 \mathrm{~Hz}, 1 \mathrm{H}), 0.98(\mathrm{~d}, \mathrm{~J}=7.7 \mathrm{~Hz}, 3 \mathrm{H}) ;{ }^{13} \mathrm{C} \mathrm{NMR}(75$ $\left.\mathrm{MHz}, \mathrm{CDCl}_{3}\right) \delta 171.5,133.0,131.2,129.6,129.3,127.2,126.9,73.3,50.3,9.1$; HRMS $\mathrm{m} / z$ calcd for $\mathrm{C}_{10} \mathrm{H}_{9} \mathrm{ClO}_{2}$ : 196.0291; found: 196.0299 .

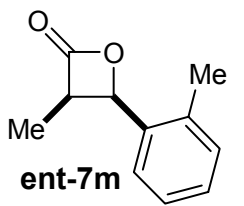

(3R, 4R)-3-Methyl-4-ortho-tolyloxetan-2-one (ent-7m): The General Procedure was followed employing $40 \mathrm{mg}$ of TMS $q(0.10 \mathrm{mmol}, 10 \mathrm{~mol} \%), 212 \mathrm{mg} \mathrm{\textrm {LiClO } _ { 4 }}(2.0 \mathrm{mmol}, 200$ mol\%) and $116 \mu \mathrm{L}$ of 2-methylbenzaldehyde $(1.0 \mathrm{mmol})$ at a reaction temperature of -40 ${ }^{\circ} \mathrm{C}$. Purification by flash chromatography (10\% ethyl acetate in hexane) gave $134 \mathrm{mg}(76 \%)$ of the title compound as colorless oil. Separation of enantiomers by chiral HPLC [Daicel Chiracel $^{\mathrm{TM}}$ OD-H column, flow rate $0.5 \mathrm{~mL} / \mathrm{min}, 4 \%{ }^{i} \mathrm{PrOH}, 96 \%$ hexane, $\mathrm{T}_{\mathrm{r}} 18.1 \mathrm{~min}(3 R, 4 R), 19.1 \mathrm{~min}(3 S$, $4 S)]$ provided only one enantiomer $(3 R, 4 R)\left(>99 \%\right.$ ee). $[\alpha]_{\mathrm{D}}+202\left(c 1.49, \mathrm{CHCl}_{3}\right)$; IR (thin film): 2978, 1829,1493, 1260 $\mathrm{cm}^{-1}$; ${ }^{1} \mathrm{H}$ NMR $\left(300 \mathrm{MHz}, \mathrm{CDCl}_{3}\right) \delta$ 7.48-7.45 (m, 1H), 7.31-7.20 (m, 3H), $5.78(\mathrm{~d}, \mathrm{~J}=6.3 \mathrm{~Hz}$, $1 \mathrm{H}), 4.08(\mathrm{qd}, \mathrm{J}=7.7,6.3 \mathrm{~Hz}, 1 \mathrm{H}), 2.22(\mathrm{~s}, 3 \mathrm{H}), 0.91(\mathrm{~d}, \mathrm{~J}=7.7 \mathrm{~Hz}, 3 \mathrm{H}) ;{ }^{13} \mathrm{C} \mathrm{NMR}\left(75 \mathrm{MHz}, \mathrm{CDCl}_{3}\right) \delta 171.9$, 133.8, 132.9, 130.2, 128.2, 126.3, 124.9, 73.7, 49.5, 18.7, 9.2; HRMS $m / z$ calcd for $\mathrm{C}_{11} \mathrm{H}_{12} \mathrm{O}_{2}$ : 176.0837; found: 176.0831 .

Stereochemical proofs for $\beta$-lactones: The absolute configuration of $\beta$-lactones ent-7h and $\mathbf{7 i}$ was established by conversion to the corresponding methyl esters $\mathbf{8 h}$ and $\mathbf{8 i}$ using the published procedure $\left(\mathrm{La}\left(\mathrm{O}^{t} \mathrm{Bu}\right)_{3}, \mathrm{MeOH}\right)^{7}$ and correlating the ester's specific rotations to those of authentic samples of known configuration: 8h $[\alpha]_{\mathrm{D}}^{23}=-21(2 R, 3 S)\left(c 0.58, \mathrm{CHCl}_{3}\right)\left[\right.$ lit $[\alpha]_{\mathrm{D}}{ }^{23}=+16(2 S, 3 R)(c 0.48$, $\left.\left.\mathrm{CHCl}_{3}\right)\right] ;{ }^{8} 8 \mathbf{i}[\alpha]_{\mathrm{D}}{ }^{23}=+5.2(2 S, 3 R)\left(c 1.64, \mathrm{CHCl}_{3}\right)\left[\right.$ lit $\left.[\alpha]_{\mathrm{D}}{ }^{23}=-5.5(2 R, 3 S)\left(c 0.3, \mathrm{CHCl}_{3}\right)\right] .{ }^{9}$ The configuration of lactone ent-7 $\mathbf{j}$ was established similarly by conversion to the corresponding ethyl ester $\mathbf{8 j}$ according to the literature procedure $\left[\mathrm{La}\left(\mathrm{O}^{t} \mathrm{Bu}\right)_{3}, \mathrm{EtOH}\right]: \mathbf{8 j}[\alpha]_{\mathrm{D}}{ }^{23}=+20.6(2 R, 3 R)(c 1.07$, $\left.\mathrm{CHCl}_{3}\right)\left[\right.$ lit $\left.[\alpha]_{\mathrm{D}}{ }^{23}=-22.0(2 S, 3 S)\left(c 0.87, \mathrm{CHCl}_{3}\right)\right] .{ }^{10}$ The absolute configuration of $\beta$-lactone ent-7e

7 Nelson, S. G.; Wan. Z.; Peelen, T. J.; Spencer, K. L. Tetrahedron Lett. 1999, 40, 6535-6540.

8 Ghosh, A. K.; Kim, J.-H. Tetrahedron Lett. 2002, 43, 5621-5624.

9 Carda, M.; Murga, J.; Falomir, E.; Gonzalez, F.; Marco, J. A. Tetrahedron: Asymm. 2000, 11, 3211-3220.

10 Hena, M. A.; Terauchi, S.; Kim, C.-S.; Horiike, M.; Kiyooka, S.-i. Tetrahedron: Asymm. 1998, 9, 1883-1890. 
was established similarly by conversion to the corresponding $N$-methoxy- $N$-methyl amide 8e using the published procedure $\left[\mathrm{MeO}(\mathrm{Me}) \mathrm{NH}_{2} \mathrm{Cl}, \mathrm{AlMe}_{3}\right]:{ }^{11}[\alpha]_{\mathrm{D}}{ }^{24}=-28.8(2 R, 3 S)\left(c 1.56, \mathrm{CHCl}_{3}\right)\left[\mathrm{lit}[\alpha]_{\mathrm{D}}{ }^{25}=\right.$ $+24.4(2 S, 3 R)\left(c\right.$ 1.33, $\left.\left.\mathrm{CHCl}_{3}\right)\right] .{ }^{12}$ The configuration of the remaining $\beta$-lactones 7 and ent-7 was assigned by analogy to these determinations.
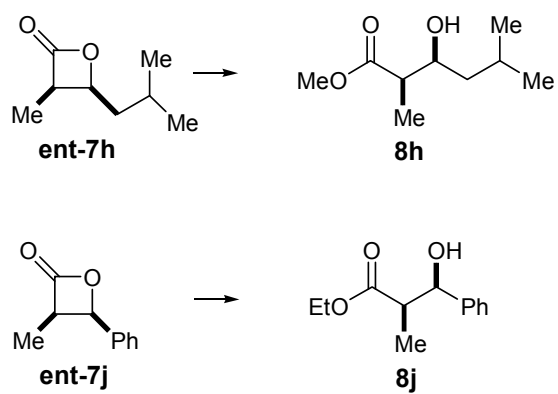

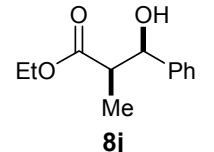

$8 \mathbf{j}$
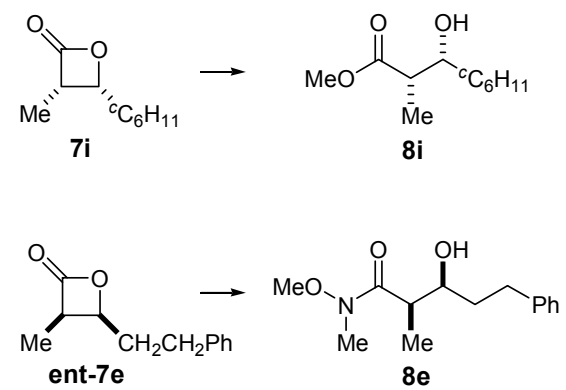

11 Nelson, S. G.; Cheung, W. S.; Kassick, A. J. Hilfiker, M. A. J. Am. Chem. Soc. 2002, 124, 13654-13655.

12 Supporting Information for Marshall, J. A.; Adams, N. D. J. Org. Chem. 1999, 64, 5201-5204. 

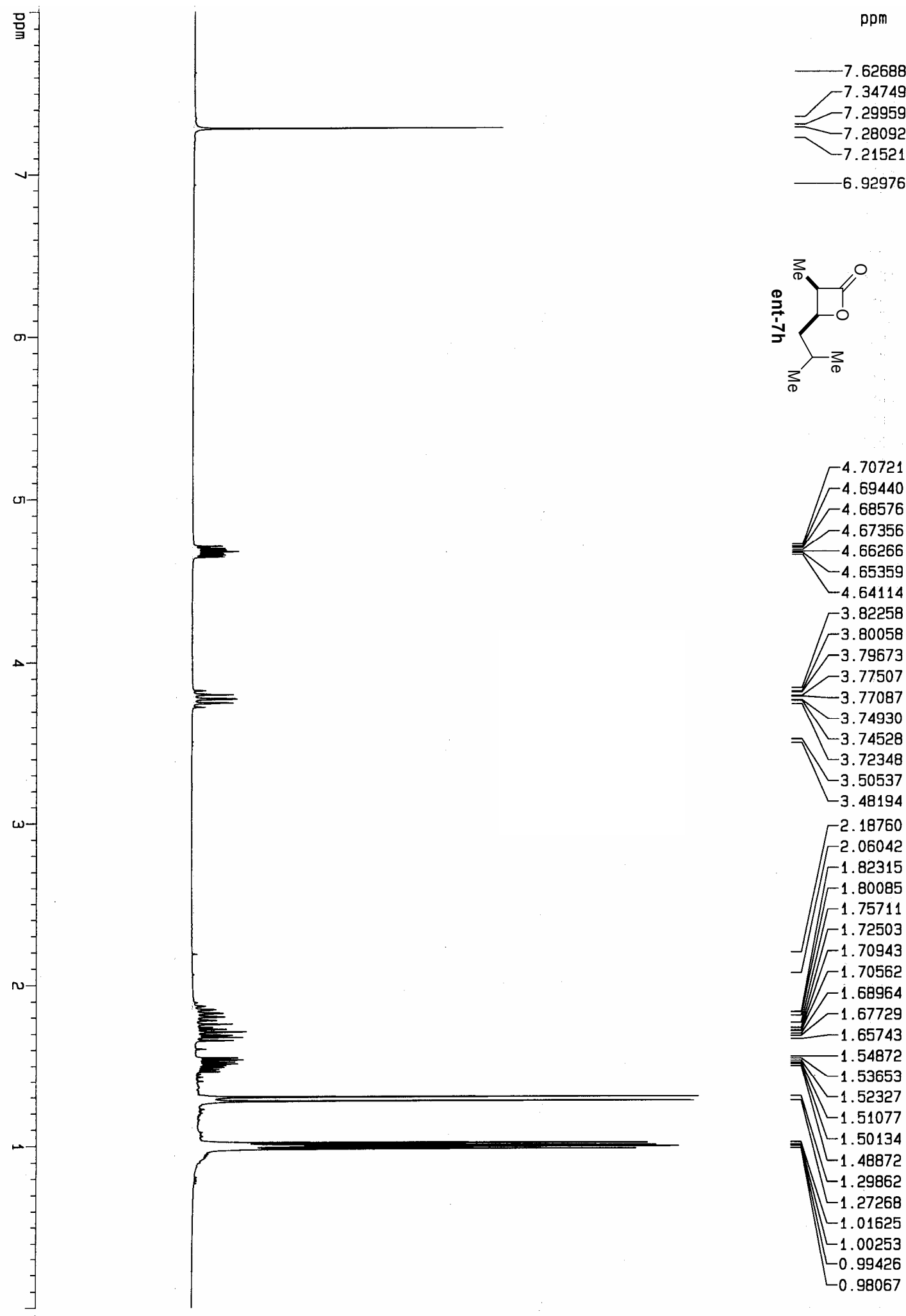

$-3.82258$

3.80058

$-3.79673$

$-3.77507$

$-3.77087$

$-3.74330$

ר -3.74528

$-3.72348$

$-3.50537$

$-3.48194$

$-2.18760$

$-2.06042$

$-1.82315$

$-1.80085$

$-1.75711$

$-1.72503$

$-1.70943$

] -1.70562

$-1.68964$

캐 -1.67729

$-1.65743$

五 1.54872

$-1.53653$

$\sqrt{-1.52327}$

$-1.51077$

a $L_{1.50134}$

$L_{1.48872}$

$-1.29862$

$-1.27268$

$-1.01625$

$L_{1.00253}$

$\leftarrow 0.99426$

$\leftarrow_{0.98067}$ 

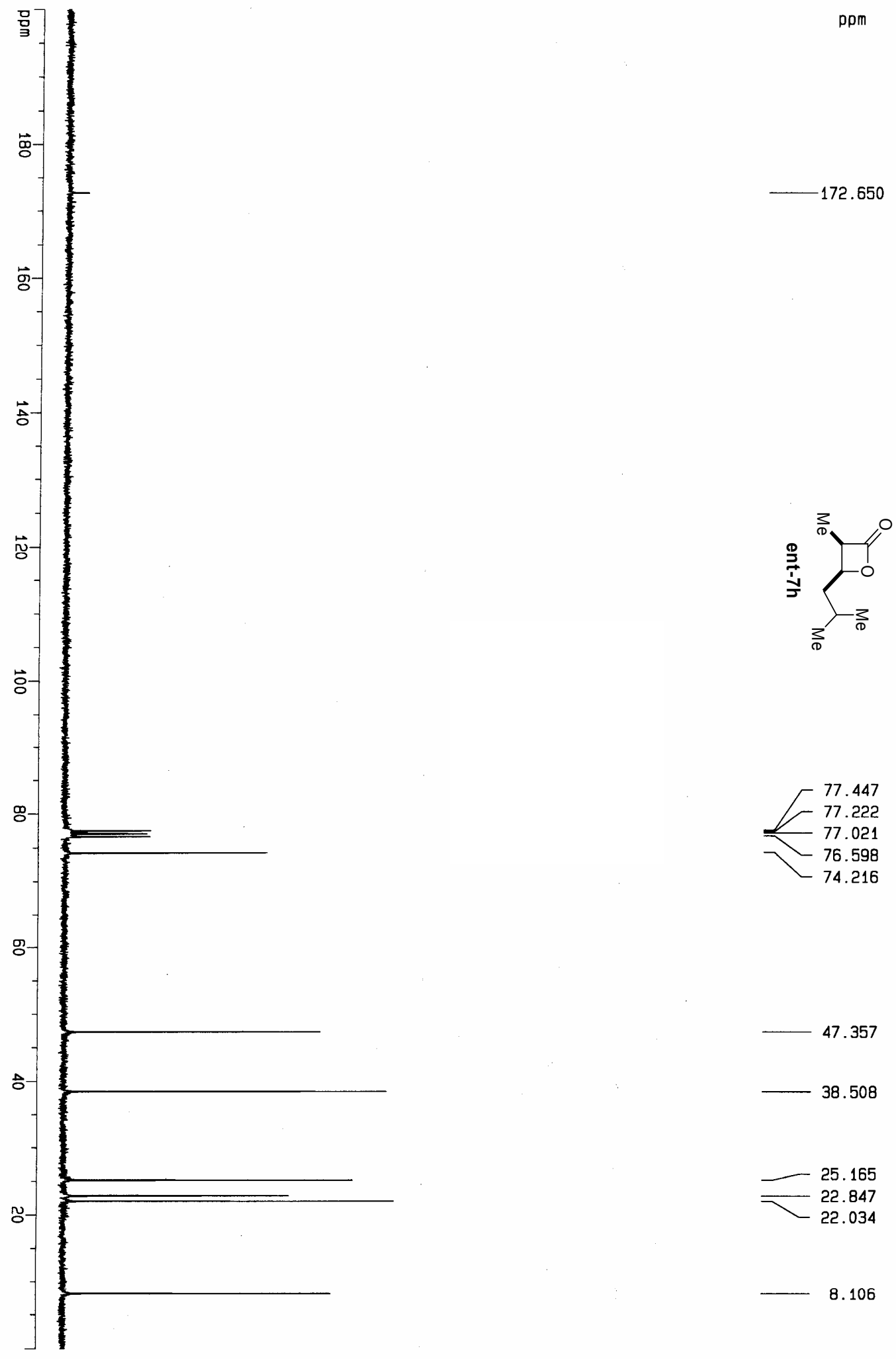


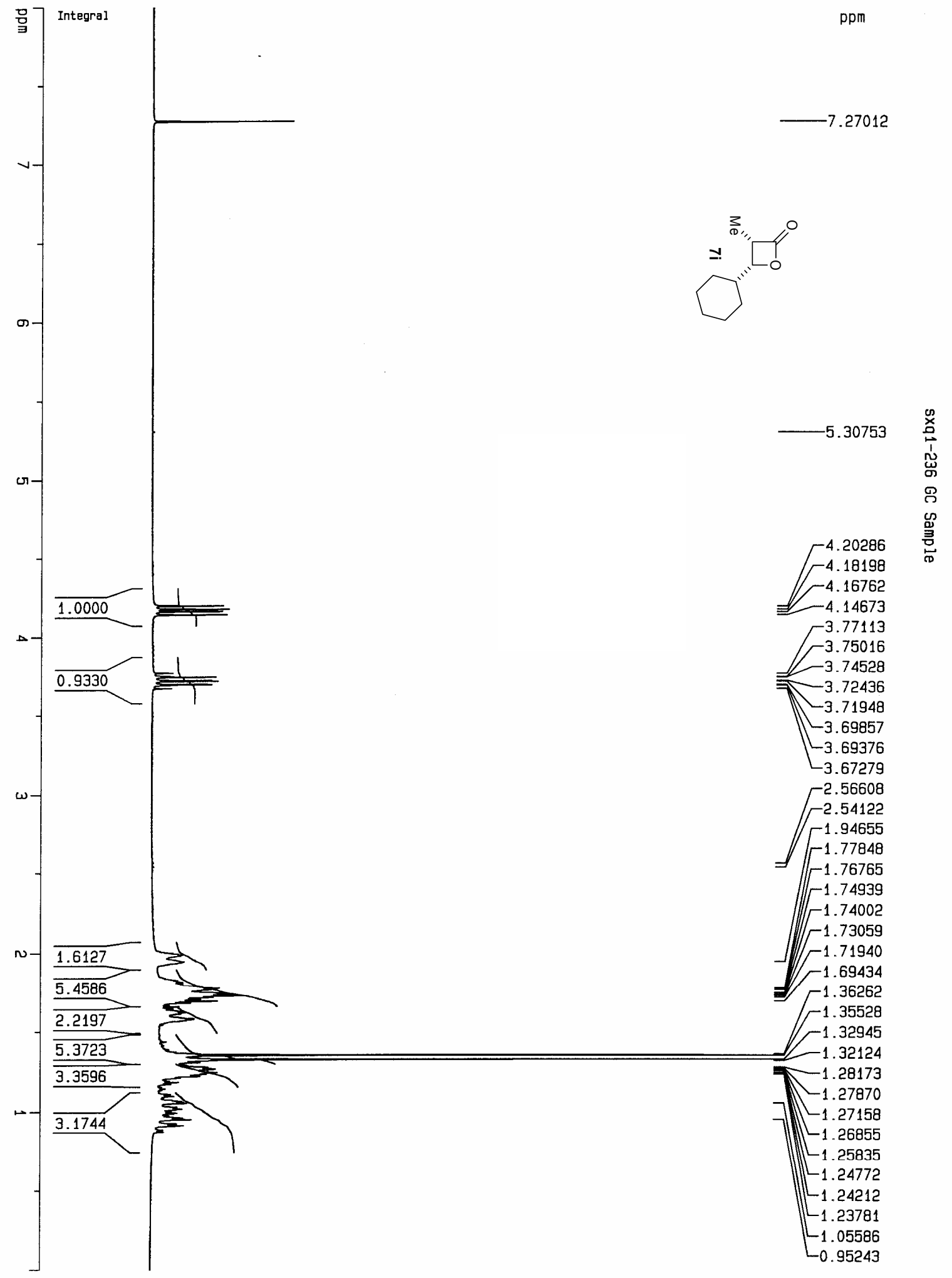




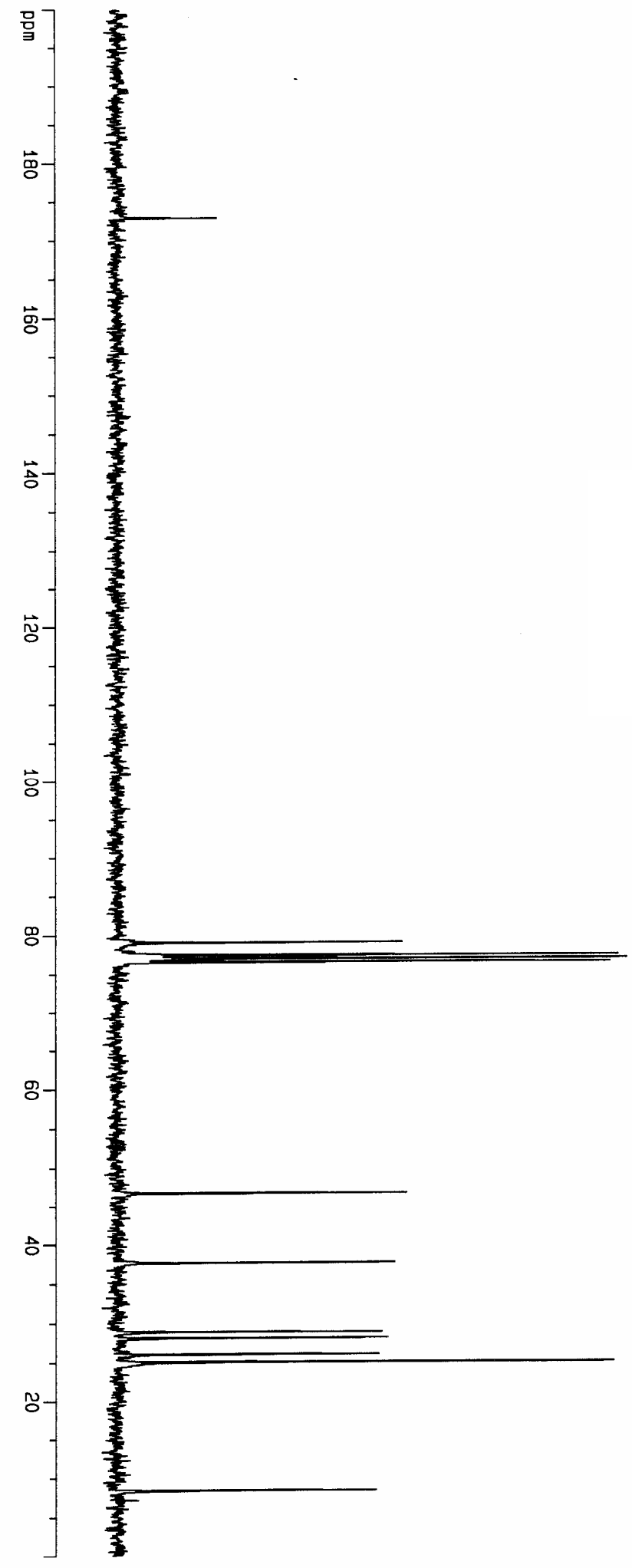

79.047

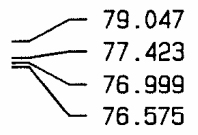

$-46.761$

$-37.708$

28.888

28.109

26.022

25.049

8.429 

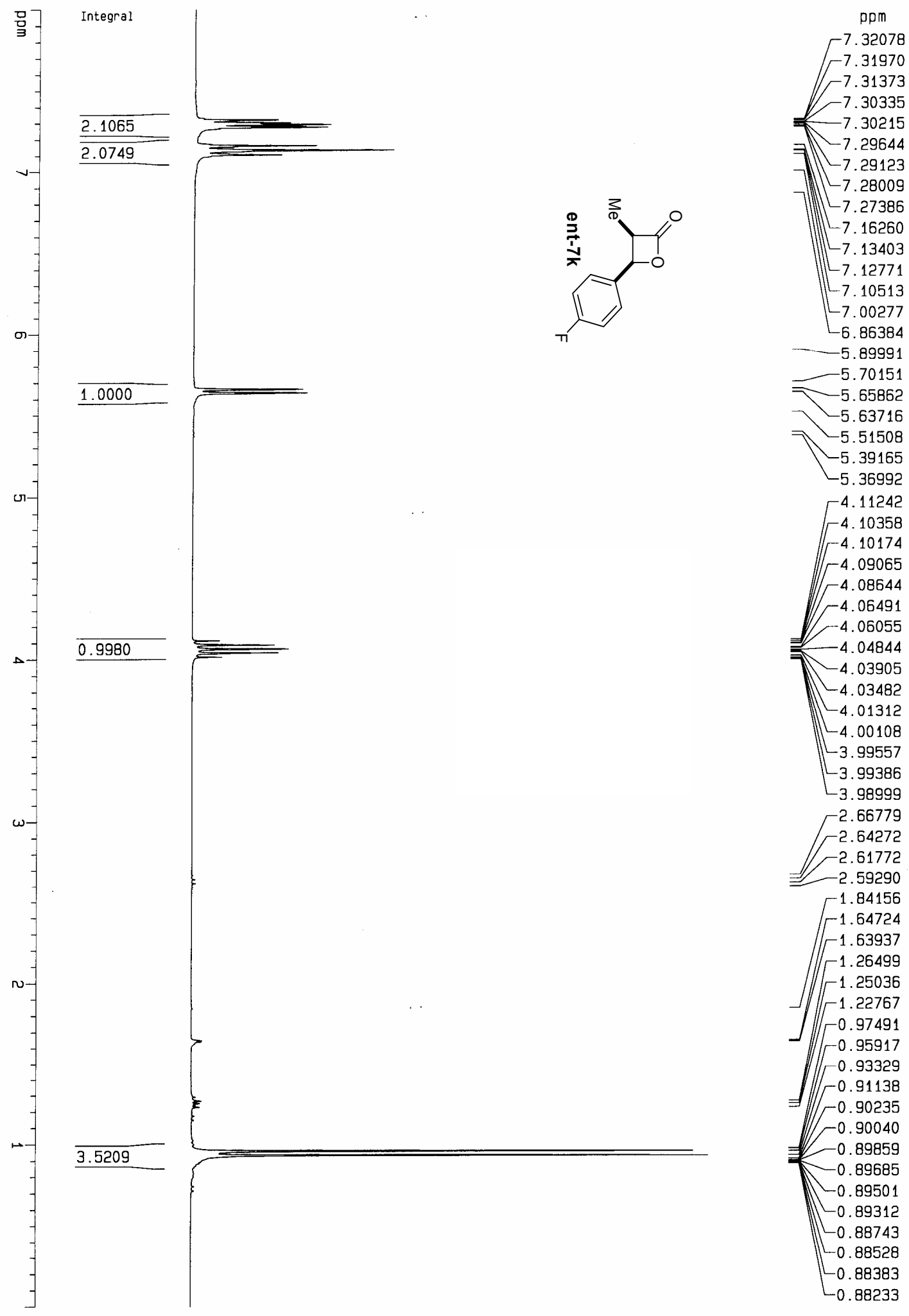


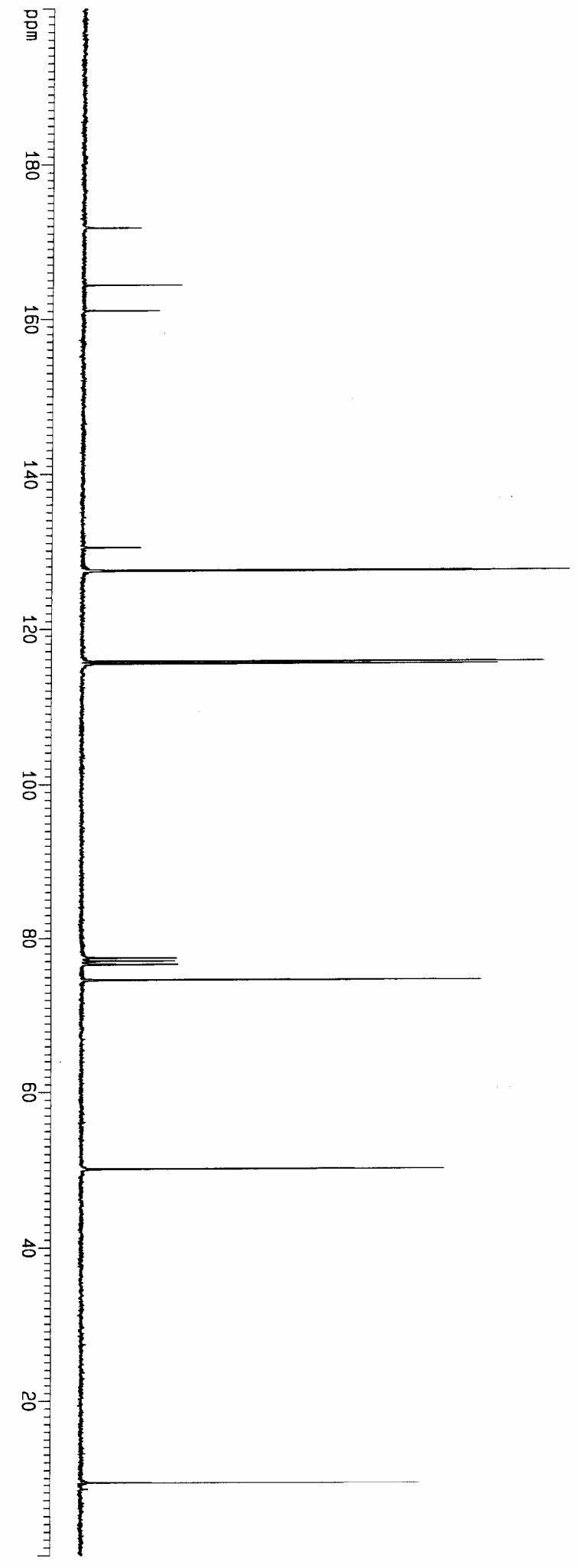
ppm

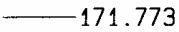

-164.312
$-\quad 161.032$
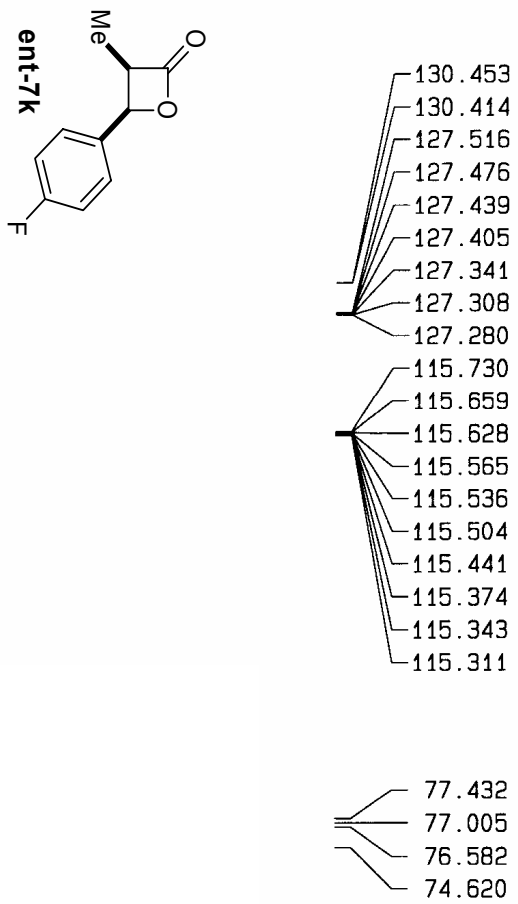

50.119

9. 356 


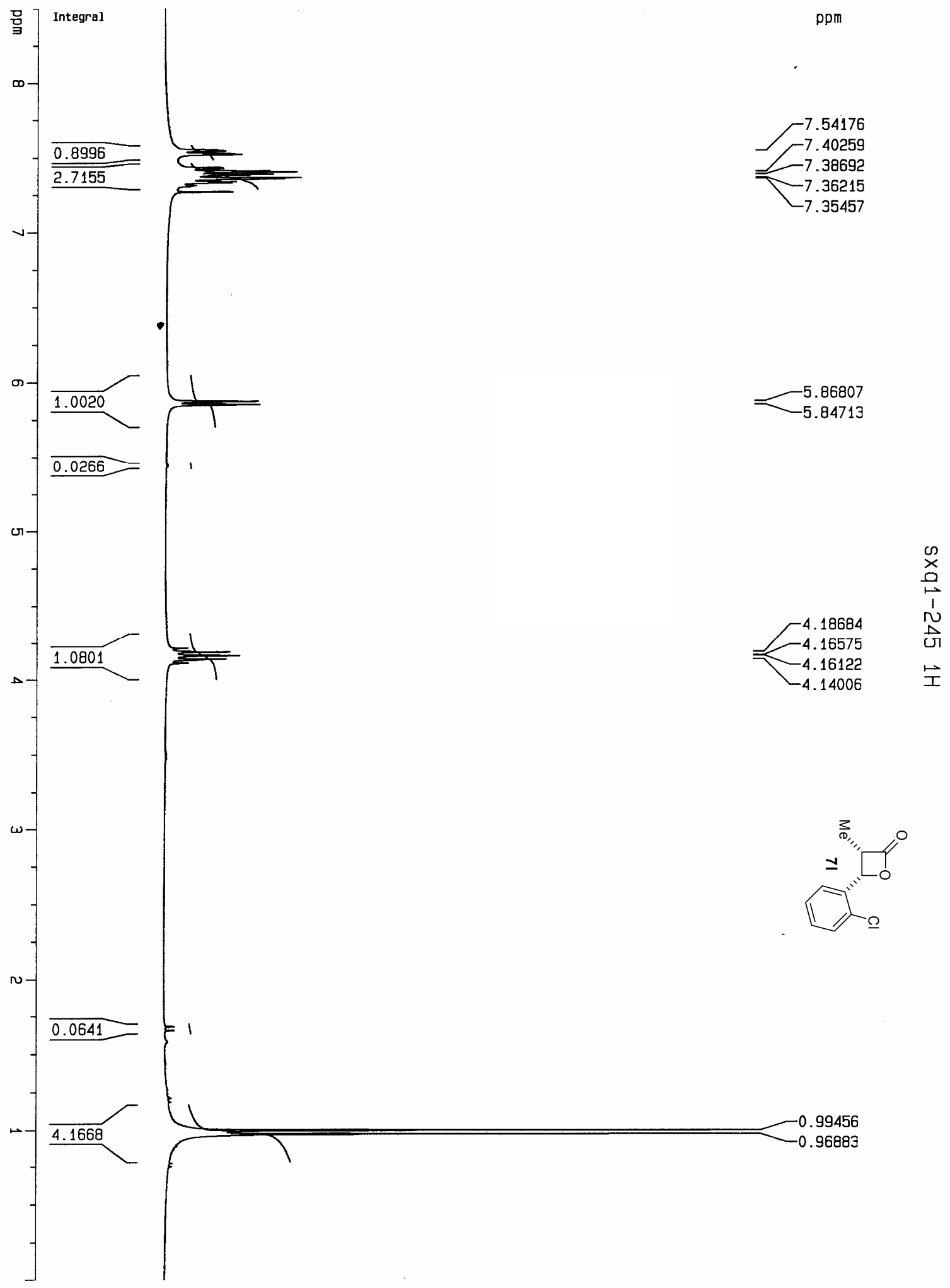




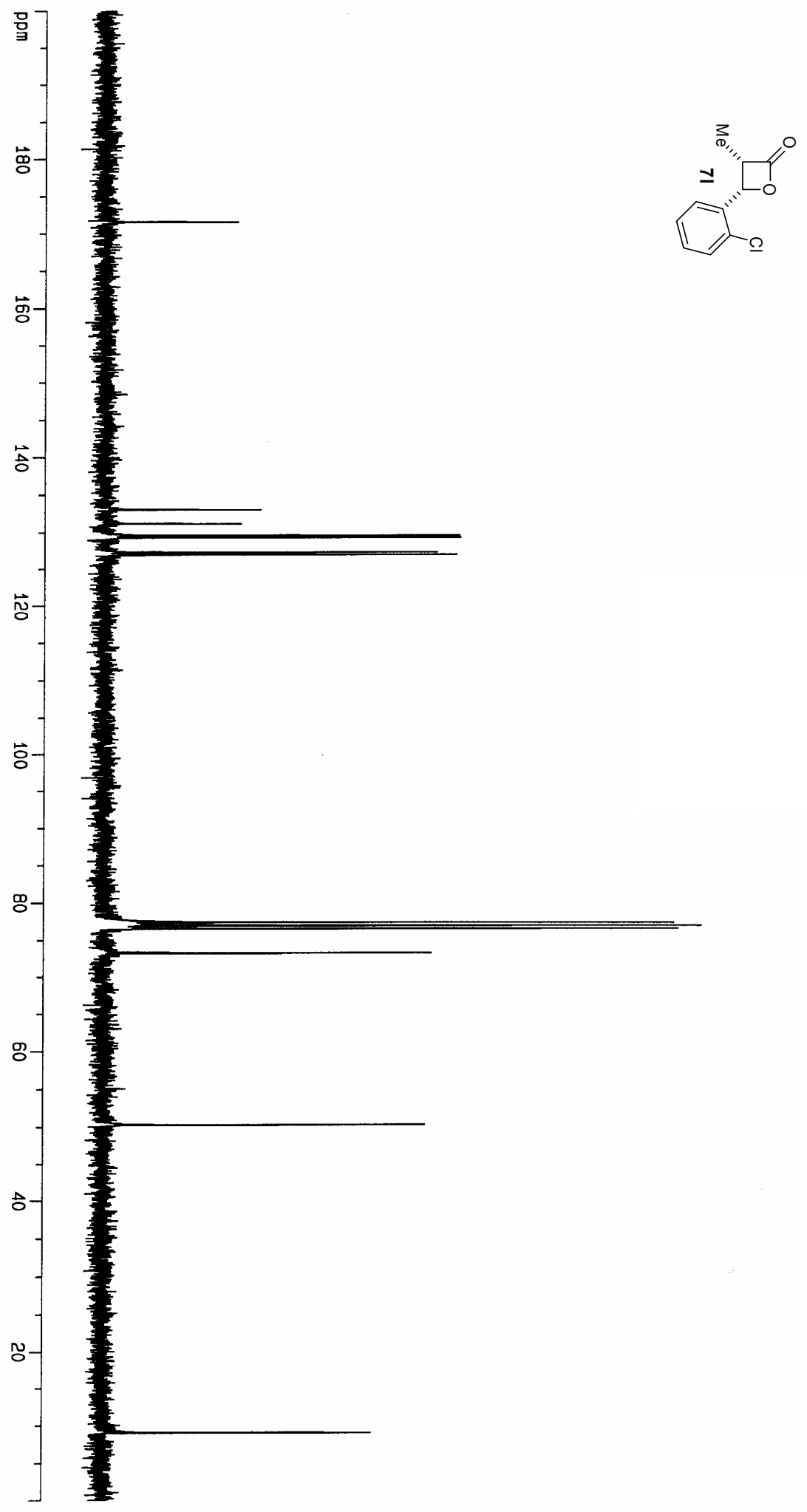

ppm
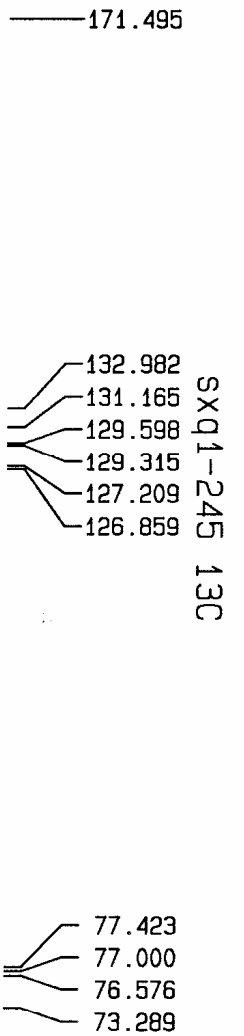

50.291

9.087 

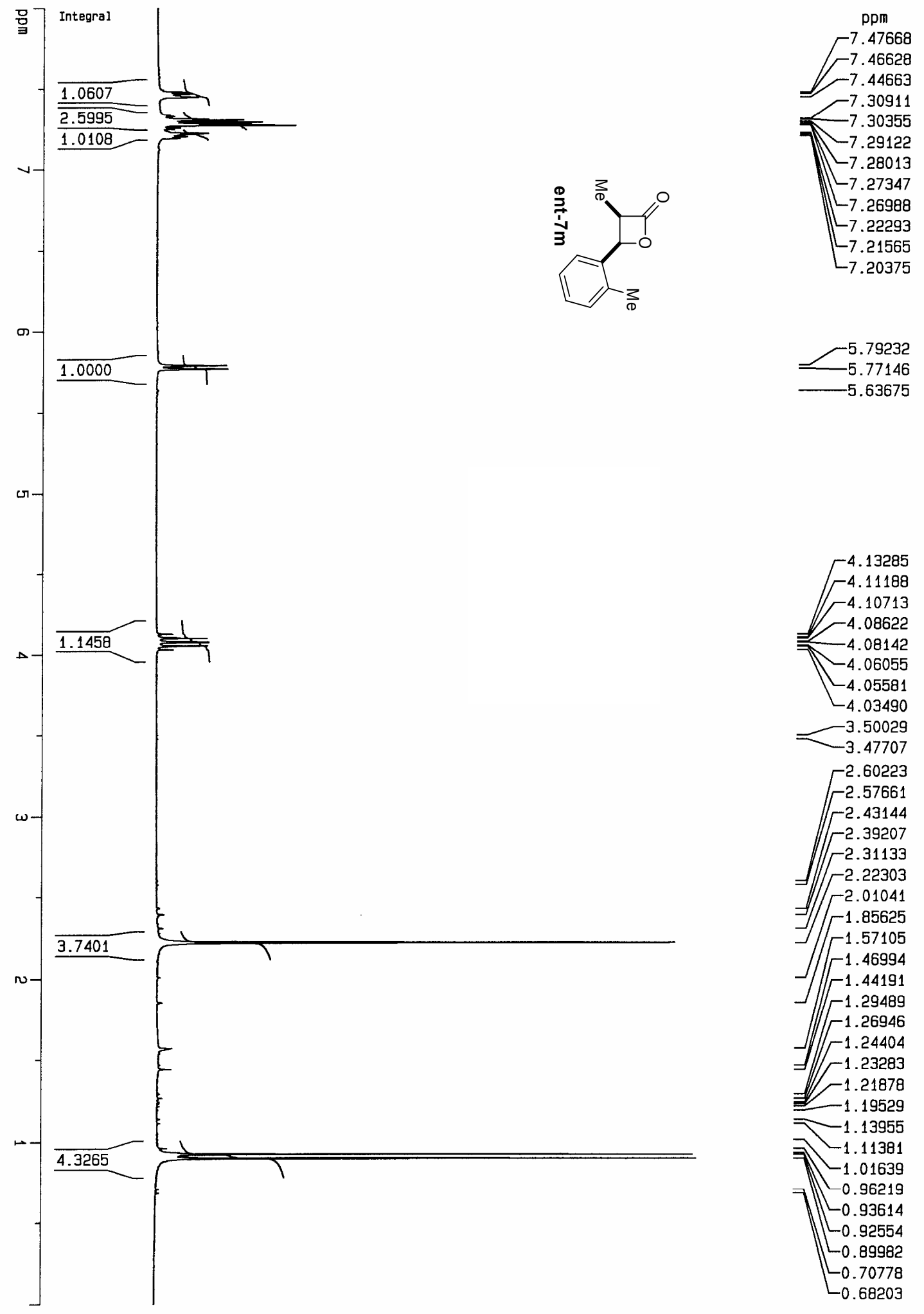

4.13285

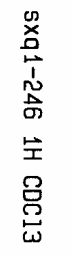

$\tau^{4.10713}$

4.10713
4.08622
4.08142

-4.06055
-4.05581

-4.05581
-4.03490

-3.50029
-3.47707

-2.60223

$-2.57661$

2.43144

-2.39207

-2.31133

$=-2.22303$

-2.01041

$\exists / \Gamma^{-1.85625}$

] 1.57105

$\sqrt{1.46994}$

$-1.44191$

]$/ \Gamma^{1.29489}$

]$\Gamma^{1.24404}$

$=1.23283$

$=\begin{array}{r}1.21878 \\ =1.19529\end{array}$

$-1.13955$

⿹ -1.11381

$7-1.01639$

| -0.93614

$-0.92554$

Lo.89982

$L_{0.70778}$

$L_{0.68203}$ 

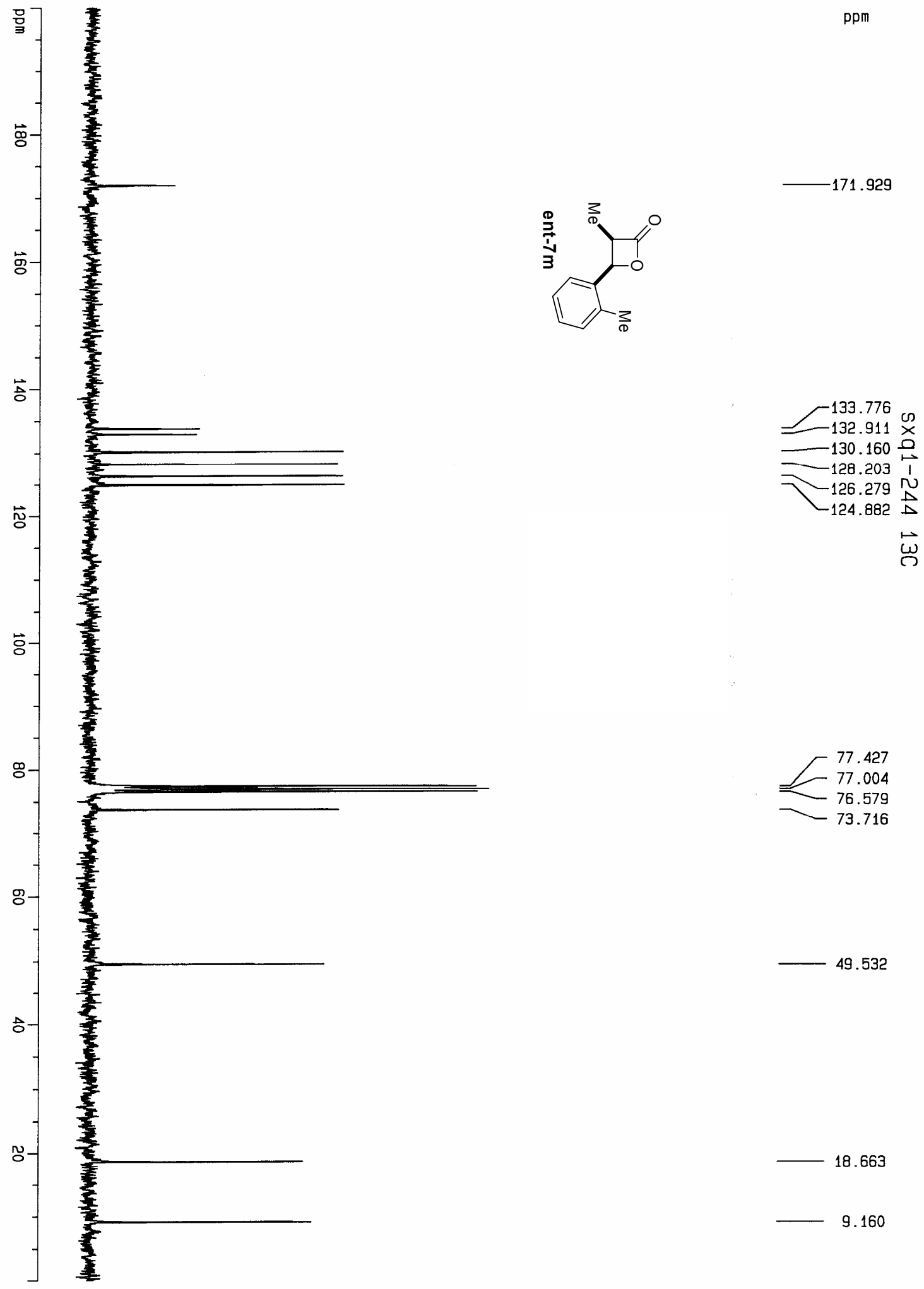

49.532

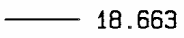

9.160 